\title{
Evaluación de competencias quirúrgicas de los egresados del Programa de Formación en Otorrinolaringología de la Universidad de Chile
}

\author{
Surgical skills evaluation of the graduates from the training program \\ in Otorhinolaryngology of the University of Chile
}

\author{
Felipe Gauna P1, Yerco Goldman P1, Mariela Torrente A².
}

\begin{abstract}
RESUMEN
Introducción: En Chile, la especialidad de otorrinolaringología (ORL) es impartida por 4 universidades. La acreditación de los programas de formación de especialistas está a cargo de la Agencia Acreditadora de Programas de Posgrado de Especialidades en Medicina y Centros Formadores de Médicos Especialistas (APICE). Para la especialidad de ORL se propone una lista de cirugías y un número mínimo a realizar durante sus años de formación, esta recomendación surgió del análisis de un grupo de expertos.

Objetivo: Evaluar número y tipos de cirugías realizadas por egresados del Programa de Formación en ORL de la Universidad de Chile y comparar estos resultados con los criterios recomendados por APICE.

Material y método: Se revisaron los portafolios quirúrgicos de los egresados del programa de ORL entre los años 2006-2016 entregados en la Escuela de Posgrado de la Universidad de Chile. Se registró nombre de cirugía, rol en la cirugía (cirujano o ayudante), unidad académica, año de residencia en que se realizó y año de egreso.

Resultados: Se revisaron 77 portafolios, de los cuales se excluyeron 11 (1 por no tener examen rendido, 1 por entregar datos mal registrados y 9 por no especificar rol en cirugía), por lo que se tabularon 66. Ninguno de los egresados cumple con las recomendaciones APICE.

Conclusiones: La Universidad de Chile posee el programa de formación en ORL más antiguo y que aporta la mayor cantidad de egresados anualmente, sin embargo ninguno de éstos ha cumplido totalmente las recomendaciones de la agencia acreditadora APICE durante los últimos 10 años. Existen procedimientos frecuentes en la especialidad no incluidos en las recomendaciones.
\end{abstract}

Palabras clave: Curva de aprendizaje, habilidades, destrezas, programa de formación.

\section{ABSTRACT}

Introduction: In Chile there are 4 universities with postgraduate programs in Otorhinolaryngology. The national organism that regulates postgraduate education in Medicine

\footnotetext{
1 Alumno Escuela de Medicina, Facultad de Medicina Universidad de Chile.

2 Médico del Departamento de Otorrinolaringología, Facultad de Medicina Universidad de Chile.

Recibido el 9 de octubre, 2017. Aceptado el 22 de octubre, 2017.
} 
(APICE) recommends a minimal number of surgeries to be performed during the training, these suggestions were developed by a group of experts.

Aim: The objective of this paper is to explore the accomplishment of these minimal number of surgeries by the graduates of the Otorhinolaryngology Program from Universidad de Chile in the last 10 years.

Material and method: The surgical portafolio from the students graduated between 2006 and 2016 were reviewed. The following data was registered: year of completion of the program, surgery, kind of participation (surgeon or not), and academic location.

Results: In the period 77 physicians completed the program, eleven of them were excluded. The total analysis of 66 portafolios revealed that none of them accomplished a $100 \%$ of the minimal requirements suggested by APICE.

Conclusions: None of the graduates from the Otorhinolaryngology Program from Universidad de Chile in the last 10 years has fulfilled in 100\% the requirements.

Key words: Surgical competence, residents, accreditation.

\section{INTRODUCCIÓN}

La especialidad de otorrinolaringología incorpora en su actividad diversos procedimientos quirúrgicos. Cada uno de ellos requiere un grado de competencia que permita maximizar los beneficios y minimizar los riesgos para los pacientes. En la literatura existen algunas publicaciones que hacen referencia a determinados procedimientos tales como estapedostomía ${ }^{1}$ o cirugía endoscópica rinosinusal $^{2}$. El número de cirugías necesario para lograr un grado óptimo de competencia es relevante para el proceso formativo ya que este análisis debiera incorporarse en la planificación del curriculum.

En los programas de formación de otros países existe información variable sobre el número y tipo de cirugías que deben realizar los residentes durante su período de estudio. En algunos programas solo se nombra el tipo de cirugía en las que deben participar, en otros el número a realizar de forma general o por año y en otros no se especifica el rol que deben tener en dicha participación (cirujano 0 ayudante) $)^{3-10}$.

En Chile, la formación de especialistas en otorrinolaringología (ORL) es impartida por 4 universidades: Pontificia Universidad Católica de Chile, Universidad de Concepción, Universidad de Valparaíso y Universidad de Chile. La formación teórica está a cargo de la Sociedad Chilena de Otorrinolaringología y Cirugía de Cabeza y Cuello (SOCHIORL), mediante un curso de 2 años de duración, que se imparte de igual forma para to- das las universidades. Por otro lado, la formación práctica depende de cada centro de formación, por lo tanto, está sujeta a diversos factores, tales como: disponibilidad de pabellón, epidemiología local, disponibilidad de tutores, tecnología e infraestructura del centro, etc. Desde el año 2008 en Chile la acreditación de programas de formación en especialidades médicas está a cargo de la Agencia Acreditadora de Programas de Posgrado de Especialidades en Medicina y Centros Formadores de Médicos Especialistas (APICE). En relación a los requisitos para desarrollar un programa de formación en ORL, APICE recomienda una lista con el número de cirugías en las que cada residente debe participar como cirujano por cada año durante su formación y certificar esto al término de sus estudios (Tabla 1).

Esta recomendación de APICE tiene su origen en la opinión de un grupo de expertos designados por la SOCHIORL.

El Programa de Formación en Otorrinolaringología de la Universidad de Chile cuenta con un curriculum basado en competencias con responsabilidades progresivas, sin embargo, no hay una exigencia del número mínimo de cirugías que debe realizar un alumno antes de egresar. Tanto la Pontificia Universidad Católica de Chile como la Universidad de Valparaíso incluyen dentro de su programa de formación una lista con el número de cirugías en las que se espera que participe el residente tanto como ayudante 0 como cirujano $0^{11,12}$.

A raíz de la necesidad de elaborar criterios de competencia en la adquisición de destrezas quirúr- 
Tabla 1. Cirugías recomendadas por APICE para cada año del programa de formación

\begin{tabular}{|lcccr|}
\hline Cirugía & Primer año & Segundo año & Tercer año & Total \\
\hline Traqueotomía & 2 & 4 & 6 & 12 \\
Punción transtimpánica & 10 & 20 & 20 & 50 \\
Timpanoplastía & 0 & 2 & 6 & 8 \\
Vaciamiento mastoideo & 0 & 2 & 6 & 8 \\
Adenoidectomía & 10 & 20 & 20 & 50 \\
Amigdalectomía & 40 & 120 & 240 \\
Drenaje absceso periamigdalino & 1 & 3 & 10 & 15 \\
Septoplastías & 1 & 2 & 6 & 10 \\
Turbinectomías & 0 & 20 & 25 & 6 \\
Reducción de fracturas nasales & 10 & 5 & 10 & 55 \\
Microlaringoscopías directas & 0 & & & 15 \\
\hline
\end{tabular}

gicas nos planteamos la pregunta de si los egresados de la Universidad de Chile cumplen con las recomendaciones de APICE. La hipótesis de trabajo es que sí se cumplen dichas recomendaciones.

\section{OBJETIVO}

El objetivo del presente trabajo es evaluar el número y tipo de cirugías que realizan los residentes egresados del programa de formación en ORL de la Universidad de Chile y comparar los resultados con Ios requisitos que recomienda APICE. Como objetivos secundarios se pretende analizar el cumplimiento de la responsabilidad progresiva y determinar si hay diferencias entre los centros formadores (unidades académicas) que colaboran con el Programa.

\section{MATERIAL Y MÉTODO}

Se revisaron los portafolios quirúrgicos entregados por cada residente en la Escuela de Posgrado de la Facultad de Medicina de la Universidad de Chile al final de su proceso formativo entre los años 2006 y 2016. Criterios de exclusión: no rendición de examen de grado e información incompleta. Se registró nombre de la cirugía, unidad académica, año de egreso, año de residencia en el que se realizó la cirugía y rol que tuvo en ésta, omitiendo las participaciones como $2^{\circ} 03^{\text {er }}$ ayudante.
Los alumnos reportan su portafolio quirúrgico en base a la codificación de procedimientos de FONASA. En dicho código no se hace diferencia para los procedimientos de amigdalectomía con 0 sin adenoidectomía, y lo mismo ocurre con septoplastía con o sin rinoplastía. Por esta razón para los efectos de esta comunicación se dejó como indicador único "amigdalectomía" y "septoplastía".

\section{Análisis estadístico}

Se calcularon promedios, medianas, desviación estándar, máximos y mínimos para cada grupo. Se utilizó el test estadístico $U$ de Mann-Whitney para dos muestras no pareadas, y Kruskal Wallis para múltiples muestras no pareadas. $p<0,05$.

\section{RESULTADOS}

Durante el período de análisis egresaron 77 alumnos. Uno de ellos no rindió examen de grado, y 10 entregaron datos incompletos (mal registro, sin especificar rol en la cirugía). Finalmente se revisaron y tabularon los portafolios de 66 egresados. De ellos 16 no especificaban el año de residencia en el que se realizó la cirugía, por lo que para el análisis por año de residencia solo se consideraron los 50 que sí especificaron este dato.

Se registró un total de 32.246 cirugías, de las cuales 25.168 (78\% del total) correspondían a las 
cirugías trazadoras de APICE. Los residentes participaron como cirujano en 19.084 procedimientos (59\% del total). En promedio cada alumno realizó 289 procedimientos como primer cirujano (Mediana =298; DS =99; $\operatorname{Max}=508 ;$ Min =96) y 206 como ayudante (Mediana $=195$; DS =74; Máx =449; Min =99). En la Tabla 2 se observa la distribución de las cirugías más frecuentes registradas.

Ninguno de los alumnos egresados del programa en los últimos 10 años cumplió con el 100\% de las recomendaciones de APICE (Tabla 3).

El promedio de cumplimiento es de $37,3 \%$ (Mediana =36,4\%; DS =15,3\%, Máx =72,7\%; Min $=9,2 \%$ ), es decir de los 11 procedimientos propuestos por APICE, se cumple en promedio con $37,3 \%$ de éstos. Al analizar por procedimiento, se observa una variación entre $0 \%$ y $73 \%$ de cumplimiento. Los procedimientos con $0 \%$ de cumplimiento corresponden al drenaje de absceso periamigdalino y amigdalectomía. Entre las cirugías que realizan los egresados como primer cirujano y no están incluidas en las recomendaciones de APICE destacan los procedimientos de cirugía endoscópica rinosinusal (Tabla 2). Las cirugías que presentan mayor divergencia con respecto a lo recomendado por APICE, son las punciones transtimpánicas y amigdalectomía.

Al analizar la oportunidad quirúrgica de los alumnos de acuerdo a su unidad académica principal se observa que no hay una distribución homogénea para la mayoría de los procedimientos. Sólo en el caso de microlaringoscopía directa no hay diferencia significativa (Tabla 4).
Tabla 2. Número total de procedimientos realizados como cirujano por egresados del Programa de Formación en Otorrinolaringología Universidad de Chile

\begin{tabular}{|lc|}
\hline Procedimiento & Número total \\
\hline Amigdalectomía* & 6.732 \\
Punción transtimpánica & 1.842 \\
Reducción de fractura nasal & 1.702 \\
Traqueotomía & 1.192 \\
Septoplastía** & 1.125 \\
Timpanoplastía & 1.102 \\
Microlaringoscopía directa & 784 \\
Vaciamiento mastoideo & 532 \\
Cirugía endoscópica nasal & 422 \\
Electrocauterización de cornetes & 377 \\
Antrostomía del seno maxilar & 191 \\
Biopsia buco-faríngea & 190 \\
Polipectomía nasal & 141 \\
Etmoidectomía & 134 \\
Abordaje hipófisis & 113 \\
Uvulopalatoplastía & 81 \\
Sección frenillo sublingual & 66 \\
\hline
\end{tabular}

*: Incluye amigdalectomía y adenoamigdalectomía. **: Incluye septoplastía y/o rinoplastía.

En relación a la responsabilidad progresiva se evaluó para seis procedimientos. Para traqueotomía y septoplastía no hay diferencia significativa al evaluar el número de procedimientos que se realizan en los tres años de formación. Para los procedimientos de microlaoringoscopía directa, vaciamiento mastoídeo y timpanoplastía se obser-

Tabla 3. Porcentaje de cumplimiento de cirugías realizadas como primer cirujano en relación a recomendaciones de APICE. DS: desviación standard

\begin{tabular}{|lccr|}
\hline & $\begin{array}{c}\text { Recomendación } \\
\text { APICE }\end{array}$ & $\begin{array}{c}\text { Promedio } \\
\text { (DS)/mediana }\end{array}$ & $\begin{array}{c}\text { Porcentaje } \\
\text { cumplimiento }\end{array}$ \\
\hline Traqueotomía & 12 & $19(15) / 15$ & 59,1 \\
Punción transtimpánica & 50 & $26(14) / 23$ & 10,6 \\
Timpanoplastía & 8 & $15(13) / 13$ & 72,7 \\
Vaciamiento mastoídeo & 8 & $8(5) / 7$ & 45,5 \\
Amigdalectomia & 240 & $96(35) / 94$ & 0 \\
Drenaje absceso periamigdalino & 15 & $0,4(0,7) / 0$ & 0 \\
Septoplastía & 10 & $18(17) / 12$ & 62,1 \\
Turbinectomías & 6 & $5(5) / 3,5$ & 43,9 \\
Reducción fracturas nasales & 55 & $25(40) / 4$ & 21,2 \\
Microlaringoscopía directa & 15 & $12(6) / 12$ & 28,8 \\
\hline
\end{tabular}


Tabla 4. Distribución de cirugías por Unidad Académica (UA). DS: desviación standard, NS: no significativo

\begin{tabular}{|lcccrr|}
\hline Cirugía & $\begin{array}{c}\text { UA 1 } \\
\text { Promedio } \\
\text { (DS)/mediana }\end{array}$ & $\begin{array}{c}\text { UA 2 } \\
\text { Promedio } \\
\text { (DS)/mediana }\end{array}$ & $\begin{array}{c}\text { UA 3 } \\
\text { Promedio } \\
\text { (DS)/mediana }\end{array}$ & $\begin{array}{c}\text { UA 4 } \\
\text { Promedio } \\
\text { (DS)/mediana }\end{array}$ & $\begin{array}{c}\text { (Kruskal } \\
\text { Wallis) }\end{array}$ \\
\hline Traqueotomía & $6(5) / 5$ & $17(9) / 16$ & $11(6) / 10$ & $38(11) / 35$ & $<0,0001$ \\
Microlaringoscopía directa & $13(7) / 13$ & $15(7) / 13$ & $9(6) / 10$ & $10(4) / 10$ & NS \\
Septoplastía & $28(9) / 30$ & $10(4) / 11$ & $26(30) / 13$ & $8(3) / /$ & $<0,0001$ \\
Vaciamiento mastoídeo & $6(3) / 6$ & $9(4) / 9$ & $3(2) / 4$ & $11(7) / 10$ & 0,002 \\
Timpanoplastía & $8(4) / 7$ & $17(6) / 14$ & $6(6) / 5$ & $30(15) / 21$ & $<0,0001$ \\
Amigdalectomía & $99(37) / 91$ & $122(31) / 114$ & $70(30) / 77$ & $88(23) / 90$ & 0,008 \\
Punción transtimpánica & $25(13) / 20$ & $37(13) / 38$ & $17(13) / 11$ & $23(12) / 20$ & 0,007 \\
\hline
\end{tabular}

va un aumento significativo entre primero y segundo año, sin variación entre segundo y tercero. El número de amigdalectomías disminuye significativamente entre primero y tercer año.

Como análisis complementario se calculó la distribución en percentiles de algunas cirugías incluidas en la recomendación de APICE (Tabla 5). Para los procedimientos de traqueotomía, timpanoplastía y septoplastía el percentil 50 de los alumnos analizados realiza más de lo recomendado por APICE. Para los procedimientos de vaciamiento mastoídeo y microlaringoscopía directa lo recomendado por APICE equivale al percentil 75 .

\section{DISCUSIÓN}

La adquisición de destrezas quirúrgicas es indispensable en la especialidad de otorrinolaringología. Cuando un alumno en formación se enfrenta a un nuevo procedimiento se observa que los resultados mejoran con la repetición, disminuyendo las complicaciones. Esto se denomina curva de aprendizaje ${ }^{13}$. Existen pocos reportes en la literatura internacional en relación a la curva de aprendizaje de los procedimientos más frecuentes en nuestra especialidad. Laeeq y cols describen la adquisición de competencias para la nasofaringolaringoscopía flexible. El ensayo se realizó con alumnos de medicina que realizaban el procedimiento en maniquís. Para lograr competencia se necesitó un promedio de 6 ensayos (rango 2 a 17) por alumno, al intento número 14 se lograba $80 \%$ de éxito ${ }^{14}$.

Los programas de formación en otorrinolaringología en el extranjero han definido requerimientos mínimos que deben cumplir los alumnos. Por ejemplo, en Estados Unidos el alumno debe realizar un mínimo de 17 timpanoplastías, 15 vaciamientos mastoídeos, 8 rinoplastías, 40 etmoidectomías, entre otros ${ }^{3,4}$.

En Chile la institución encargada de la acreditación de programas de especialidades médicas APICE recomienda un número mínimo de cirugías

Tabla 5. Distribución de percentiles 25,50 y 75 de algunas cirugías incluidas en la recomendación de APICE

\begin{tabular}{|lcccr|}
\hline Procedimiento & Percentil 25 & Percentil 50 & Percentil 75 & APICE \\
\hline Traqueotomía & 7 & 15 & 29 & 12 \\
Punción transtimpánica & 15 & 23 & 37 & 50 \\
Timpanoplastía & 6 & 13 & 19 & 8 \\
Vaciamiento mastoídeo & 4 & 7 & 11 & 8 \\
Amigdalectomía & 72 & 94 & 114 & 240 \\
Septoplastía & 9 & 12 & 21 & 10 \\
Microlaringoscopía directa & 7 & 12 & 15 & 15 \\
\hline
\end{tabular}


específicas, en base a lo sugerido por la Sociedad Chilena de Otorrinolaringología Cirugía y Medicina de Cabeza y Cuello. Estas recomendaciones han sido incorporadas a los programas de algunos centros formadores nacionales como la Universidad Católica de Chile y la Universidad de Valparaíso. El programa de formación en otorrinolaringología de la Universidad de Chile no define un requisito de número mínimo de cirugías. Por esta razón se diseñó el presente trabajo para analizar si se cumplen las recomendaciones de APICE.

Los resultados muestran que ninguno de los egresados del programa en los últimos 10 años cumple con todas las recomendaciones de APICE. El porcentaje de logro fluctúa entre $0 \%$ y $72 \%$ dependiendo del procedimiento analizado. Existen múltiples razones que pueden explicar estos resultados. El registro de los procedimientos realizados por los residentes no tiene un método homogéneo de informe. Esto se refleja en que 10 de los 77 portafolios no pudieron ser evaluados por no informar el rol en la cirugía (cirujano 0 ayudante). En forma adicional la nominación del procedimiento depende de los códigos de FONASA lo que no permite discriminar entre septoplastía y rinoplastía por ejemplo. La cirugía endoscópica rinosinusal tampoco tiene códigos que permitan conocer claramente la competencia que está adquiriendo el alumno. Finalmente hay procedimientos como drenaje de absceso periamigdalino y reducción cerrada de fractura nasal que se realizan en pabellón o en policlínico dependiendo de la realidad de cada institución, lo cual puede afectar el registro.

El programa en la Universidad de Chile se ejecuta en cuatro instituciones (unidad académica), cada alumno es asignado a una de ellas donde permanece la mayoría del tiempo. Al analizar la cantidad de cirugías que realiza cada alumno se observa que hay diferencias significativas entre unidades. Cabe recordar que las situaciones locales en cada institución afectan directamente la cantidad y tipo de cirugías que se realizan. Por ejemplo, en los hospitales de la red pública se deben priorizar patologías con riesgo vital lo cual puede ir en desmedro de cirugías electivas como rinoplastía. Sin embargo, la administración del programa debe cautelar que los alumnos adquieran competencias en todas las áreas predefinidas. Una propuesta en este sentido es incrementar las rotaciones, en particular una vez identificadas las fortalezas y debilidades de cada unidad.

En relación a la responsabilidad progresiva los resultados muestran que esto se cumple para la mayoría de los procedimientos, en particular la principal diferencia se produce entre los residentes de primer y segundo año. También se observa una disminución de cirugías del anillo de Waldeyer en los residentes de tercer año, concordante con el concepto de que esta cirugía es de baja complejidad.

Como antecedente se dispone de dos trabajos que evalúan la actividad quirúrgica de residentes de la especialidad. En 2004, Maass y Naser realizaron una encuesta a residentes de otorrinolaringología de diversos centros formadores y midieron el rendimiento en relación al programa vigente en ese momento en la Universidad de Chile. Los autores concluyen que los alumnos no cumplen todos los objetivos prácticos, que realizan procedimientos no incluidos en el programa, y que hay diferencias sustanciales del número de cirugías realizadas por cada residente dependiendo del centro formador ${ }^{15}$. Castillo y cols en el año 2008 estudian el cumplimento del programa vigente en dicho año en la Universidad de Chile ${ }^{16}$. Los autores concluyen que los residentes cumplían solo $57,3 \%$ de los objetivos del programa. A pesar de esto, participaban en cerca del $90 \%$ de la actividad quirúrgica de ese centro y realizaban procedimientos que no estaban incluidos en el programa. APICE comienza a funcionar el año 2008, por esta razón ninguno de los trabajos mencionados evalúa el cumplimiento en relación a este estándar de acreditación. El aporte del presente trabajo está en la línea de analizar si estas recomendaciones son concordantes con la realidad de la formación en Chile, qué procedimientos se debieran incorporar y cuáles eliminar.

$\mathrm{Si}$ bien este trabajo no tiene un diseño que permita estimar la curva de aprendizaje en competencias quirúrgicas, tiene el mérito de describir la realidad de un centro formador importante en el país. Constituye un análisis inicial que permite identificar cuáles son los procedimientos que se realizan con mayor frecuencia y en qué número. Según lo descrito nos parece pertinente recomendar que se incluya el área de cirugía endoscópica rinosinusal, tanto procedimientos electivos como antrostomía maxilar y etmoidectomía, y procedi- 
mientos de urgencia como ligadura de arteria esfenopalatina y drenaje de abscesos subperiósticos. En relación al número mínimo recomendado de procedimientos proponemos utilizar el percentil 50 descrito en estos resultados (Tabla 5).

\section{CONCLUSIONES}

Ninguno de los egresados del Programa de Formación en Otorrinolaringología de la Univer-

\section{BIBLIOGRAFÍA}

1. Watson G, Byth K, da Cruz M. Outcomes in Stapedotomy Surgery: The Learning curve Redefined. Otology and Neurotology 2015; 36: 1601-3.

2. Laeeq K, Lin S, Diaz D, Lane A, Reh D, Bhatti N. Achievement of Competency in Endoscopic Sinus Surgery of Otolaryngology Residents. Laryngoscope 2013; 123: 2932-4.

3. http://www.acgme.org/Portals/0/ PFAssets/ProgramResources/288. PediatricOtolaryngologyCaseLogMinimums. pdf?ver=2015-11-06-120651-760. Consultado 1 de octubre 2017.

4. http://www.acgme.org/Portals/0/PFAssets/ ProgramResources/280_Required_Minimum Number_of_Key_Indicator_Procedures. pdf?ver=2015-11-06-120651-827. Consultado 1 de octubre de 2017.

5. Plan curricular de la especialidad de ORL, Universidad Nacional Federico Villarreal, LimaPerú, 2013. Consultado el 25-02-17 en: http:// www.unfv.edu.pe/facultades/fmhu/images/pdf/ Otorrinolaringologia.pdf

6. Programa oficial de la Especialidad de Otorrinolaringología, Ministerio de Educación y Ciencias, Madrid, España, 2007. Consultado el 25-02-17 en: http://www.unfv.edu.pe/facultades/ fmhu/images/pdf/Otorrinolaringologia.pdf

7. Programa de la residencia de ORL, Departamento de Docencia e investigación, Fundación Sanatorio Güemes, Buenas Aires, Argentina, 2015. Consultado el 25-02-17 en: http://www.fsg.org. ar/programas/2015/orl_2015.pdf sidad de Chile cumple con los requisitos planteados por APICE durante los últimos 10 años. Los autores consideran que esto es secundario a una recomendación no adecuada a la realidad de los centros formadores. Por esto se proponen nuevos estándares del número mínimo de cirugías a realizar. Hay diferencia significativa en la cantidad de cirugías que realizan los alumnos según unidad académica. Se cumple el criterio de responsabilidad progresiva para la mayoría de los procedimientos evaluados.

8. Programa de la residencia de ORL, Dirección de capacitación de profesionales de la salud, Ministerio de salud de la provincia de Buenos Aires, Argentina. Consultado el 25-02-17 en: http://www.ms.gba.gov.ar/ssps/residencias/ programas/Otorrinolaringologia.pdf

9. Programa docente de Otorrinolaringología, Clínica Universidad de Navarra, España, 2011. Consultado el 01-10-17 en: https://www.cun. es/documentos/archivos/programa-formativoresidentes-otorrinolaringologia-clinicauniversidad-navarra.pdf.

10. Logbook de residentes de Otorrinolaringología y Patología cervicofacial de la Unión Europea de médicos especialistas (UEMS). Consultado el 25-02-17 en: http://www.orluems.com/ gestor/upload/doc/SPANISH\%2OVERSION\%20 LOG\%20B00K.pdf

11. Programa de especialista en otorrinlaringología. Pontificia Universidad Católica de Chile, 2015. Consultado el 28-02-17 en: http://medicina. uc.cl/postgrado/programas-especialidades/ otorrinolaringologia.

12. Programa de especialización en Otorrinolaringología. Universidad de Valparaíso, 2015.

13. Khan N., Abboudi H., Khan SK., Dasgupta P., Ahmed $K$. Measuring the surgical 'learning curve': methods, variables and competency. BJU Int 2014; 113: 504-8.

14. Laeeq K., Pandian V., Skinner M., Masood H., Stewart C., Weatherly R, et als. Learning Curve for Competency in Flexible Laryngoscopy. Laryngoscope 2010; 120: 1950-3.

15. MaAss JC, Naser A. Encuesta de adquisición de 
destrezas quirúrgicas en el programa de formación de especialistas de Otorrinolaringología en Chile. Rev Otorrinolaringol Cir Cabeza Cuello 2004; 64: 214-22.
16. Castillo C, Ruz S, Breinbauer H, Cárcamo S. Programa quirúrgico para residentes de Otorrinolaringología: De la teoría a la práctica. Rev Otorrinolaringol Cir Cabeza Cuello 2004; 68: 229-23. ¿? 